\title{
EXPLORING THE POTENTIAL OF MULTIVARIATE ANALYSIS TO STUDY THE IMPACT OF COTTON LEAF CURL DISEASE ON YIELD TRAITS
}

\author{
Muhammad Mohsin Raza ${ }^{1, *}$, Muhammad Aslam Khan', Muhammad Yaseen², Adeela Munawar² \\ and Zarnab Sabir ${ }^{1}$
}

\author{
${ }^{1}$ Department of Plant Pathology, University of Agriculture, Faisalabad, Pakistan; \\ ${ }^{2}$ Department of Mathematics and Statistics, University of Agriculture, Faisalabad, Pakistan. \\ "Corresponding author's e-mail: mohsinuaf@live.com
}

\begin{abstract}
Cotton production in Pakistan seems adequate, but inclined to several biotic and abiotic constraints, especially Cotton Leaf Curl Virus posing a hazardous impact on cotton yield by affecting yield traits, fiber quality and health status of plants. In this study, eight Bt and four Non-Bt cotton genotypes were sown under augmented design in the experimental area of the Department of Plant Pathology, University of Agriculture, Faisalabad. Presence of virus was confirmed by using the graft inoculation technique in greenhouse while natural inoculum was also relied upon for infection under field conditions. Data encompassing plant height, number of bolls, $50 \%$ open bolls, boll weight, number of sympodial and monopodial branches were collected after disease appearance and subjected to multivariate analyses of variance which revealed that all genotypes responded distinctly to Cotton Leaf Curl Disease. Then these data were subjected to linear discriminant analysis where first two linear discriminants explained $61.05 \%$ and $23.54 \%$ variability respectively, and highlighted plant height and $50 \%$ open bolls as maximum variability explaining cotton yield traits in response to disease. Territorial map was constructed between first two linear discriminants which revealed that disease influenced plant height and 50\% open bolls of Bt cotton genotypes potentially than the Non-Bt genotypes. Moreover, data for plant height and 50\% open bolls were split and subjected to Hypothesis-error (HE) plot which depicted negative correlation between plant height and $50 \%$ open bolls and described the results in conformation with territorial map. Hence, these tools can be potentially used to study the impact of disease on crop yield traits and for selection of lines/varieties either for future breeding program or farmer field.
\end{abstract}

Keywords: Cotton viruses, yield traits, multivariate analyses, linear discriminant analysis, hypothesis-error plot territorial map.

\section{INTRODUCTION}

Cotton is an important commercial crop of global importance. Its production is the main source of income for about 100 million families (Fortucci, 2002). Due to its global importance, it is cultivated in more than 111 countries of the world (Maas, 2013). Pakistan is the $4^{\text {th }}$ largest cotton producer worldwide. Pakistan's 2015-16 cotton production is forecast at 10 million $480 \mathrm{lb}$ bales, equivalent to 2.2 million metric tons (MMT) with an area under production of 2.95 million hectares (USDA, 2016). The current status of cotton in Pakistan is prone to a number of biotic as well as abiotic factors (diseases and insect pests), among of which cotton leaf curl disease (CLCuD) is the potential threat (Briddon and Markham, 2000) which is caused by Cotton leaf-curl virus $(\mathrm{CLCuV})$ a major biotic threat to cotton production in Punjab, India (Singh et al., 2013). It appeared during 1990 at an economic proportion in Pakistan and ensued hefty yield losses during 1992-1993 (Khan and Khan, 2000).

The disease has very characteristic symptoms including leaf curling, vein thickening and darkening and enations on the veins on the underside of leaves, which frequently develop into leaf like structures (Briddon et al., 2001). Losses due to CLCuD are dependent on infectivity time and variety (Akhtar et al., 2004). The disease had adverse effect on morphological, yield and yield components and fiber quality of all cotton varieties (Ahmad et al., 2002). The pronounced damage of CLCuD is in early stages, but at later stages results in minor infections (Akhtar et al., 2004). CLCuD damage differs on various plant parts and ultimately results in reduction of yield (Ahmed, 1999). The highest reduction in seed cotton yield due to cotton leaf curl virus disease has been reported by many workers (Idris, 1990; Harrison et al., 1997; Brown, 2001; Ahmad et al., 2002). For instance, CLCuD can engender to an average reduction in plant height $(40.6 \%)$, boll weight $(33.8 \%)$, number of bolls per plant $(72.5 \%)$, ginning outturn (3.9\%), fiber length (3.4\%), fiber strength $(0.7 \%)$ and fineness in cotton cultivars (Mahmood et al., 1995).

Univariate data analysis techniques are still being used while living in the age of multivariate (Gray-McGuire et al., 2009) where it is irrational to deal the multi responses individually. For instance, Ahmad et al. (2008) used analysis of variance (ANOVA) and Fisher's protected least-significant difference (LSD) test to compare multivariate data encompassing yield 
and yield-components and fiber traits of different genotypes/varieties under different plant spacing and nitrogen fertilizer and found that CLCuV infestation varied significantly with genotypes. Similarly, (Farooq et al., 2013) employed ANOVA, LSD, T-test and Path coefficient analysis (Dewey and Lu, 1959) to determine genotypic, phenotypic correlation coefficients between seed cotton yield, earliness, fiber and yield contributing traits in 31 cotton cultivars under $\mathrm{CLCuV}$ intensive conditions. Results obtained via ANOVA when used to analyze multivariate data can be misleading. Major reasons behind the issue are increase in Type I error and dependence between response variables cannot be ignored.

Multivariate analysis of variance (MANOVA) is used in place of ANOVA to study the differences in multi responses (Bray and Maxwell, 1985). MANOVA extends the ideas and methods of univariate ANOVA in simple and straightforward ways (Friendly, 2007). The MANOVA can measure multiple dependent variables at once than ANOVA which only allows for one. The ability to measure the effects of an independent variable on multiple dependent variables is useful for comparing the effect of the independent variable in different settings (Weinfurt, 1995). Moreover, the MANOVA also increases the chance of finding the effect of an independent variable. When measuring the independent variable's effect on multiple dependent variables, one can find that there is a significant influence on one of the dependent variables, but not the others. On the other hand, using an ANOVA would have only been testing one of the dependent variables (Haase and Ellis, 1987).

Discriminant analysis can be used as a technique beyond MANOVA to assess the treatment groups differ significantly from each other. This is a group separation technique developed by Fisher (1940). Discriminants are used to see which response variables contribute significantly among group separation. Graphical representation of group centroids obtained by discriminants is used to visualize the null hypothesis of equal means. Hypothesis Error plot (HE plots) is the two dimensional plot of any type of null hypothesis of MANOVA. In the MANOVA, the HE plot shows directly how the group means differ on the two variables jointly (Friendly, 2007).
Therefore, strengths of two diverse statistical tools including Linear Discriminant Analysis (LDA) and Hypothesis-error (HE) plot were explored and found significant to determine the impact of CLCuD on yield traits (especially plant height, number of sympodial and monopodial branches, boll weight, number of bolls per plant and 50\% open boll) of different cotton genotypes.

\section{MATERIALS AND METHODS}

Collection and establishment of disease screening nursery: Twelve cotton advance lines comprising different $\mathrm{Bt}(\mathrm{VH}-$ 306, VH-307, VH-309, VH-311, VH-327, VH-335, VH-336, VH-340) and Non-Bt (VH-281, VH-289, VH-300, MNH789) were obtained from Cotton Research Institute, Vehari. Disease screening nursery was established under augmented design in the experimental area of the Department of Plant Pathology, University of Agriculture Faisalabad during 2013.The nursery was sown on $21^{\text {st }}$ March, 2013. Each test entry was planted in row to row distance $0.61 \times 0.15 \mathrm{~m}$ and plant to plant spaced $0.30 \mathrm{~m}$. The agronomic practices were followed to maintain the crop in good condition.

Inoculation: Graft inoculation technique was used for viral inoculation. CLCuVD inoculums for all graft inoculation studies were collected from naturally infected cotton plants exhibiting characteristic leaf curl symptoms of CLCuVD. The virus was transmitted by grafting infected plant branches onto healthy plants of similar genotypes, grown in earthen pots under insect-free conditions in a greenhouse. Grafting was performed using the bottle leaf grafting method as described by Akhtar et al. (2002).

Data collection: Progression of the disease based on visual symptoms was recorded on weekly basis. Ten plants per row were randomly selected and their leaves showing clear symptoms (curling, vein thickening, and enation) were observed. Cotton leaf curl virus disease (CLCuVD) ratings were assessed on 0-5 grades with the first initiation of disease symptoms (Table 1). The data were recorded according to the following disease rating scale (Mughal and Khan, 2006).

Data recording: The data regarding different yield parameters, especially plant height, number of monopodial and sympodial branches, number of bolls per plant, boll

Table 1. Disease rating scale used to determine the level of resistance or susceptibility of cotton lines to CLCuV.

\begin{tabular}{|c|c|c|}
\hline Sr. No. & Grade symptoms description & Level of resistance or susceptibility \\
\hline 0 & No symptoms & Highly resistant \\
\hline 1 & Scattered thickening of small veins & Resistant \\
\hline 2 & Thickening of all veins but no curling & Moderately resistant \\
\hline 3 & Thickening of veins and curling of leaves at the top leaves (light effect) & Moderately susceptible \\
\hline 4 & $\begin{array}{l}\text { Thickening of veins and curling of leaves on half of the plant with } \\
\text { enation (medium effect) }\end{array}$ & Susceptible \\
\hline 5 & $\begin{array}{l}\text { Thickening of veins and curling of leave (upward/downward) on the } \\
\text { entire plant and dwarfing of plant (severe effect) }\end{array}$ & Highly Susceptible \\
\hline
\end{tabular}


weight and yield losses were recorded to assess the yield losses in different Bt and Non-Bt cotton lines/varieties. Plant height was recorded from cotyledonary leaves to apical growth of plant. At maturity, the monopodial branches developed on each plant were counted in each of replication and average number of monopodials was worked out for all genotypes. Similarly, number of sympodial branches on individual plants were counted and recorded. Average number of sympodial branches was worked out for all genotypes. Number of bolls per plant were recorded from individual plants at each picking. When final picking was over, picking record was summed up and average numbers of bolls per plant were calculated. In case of boll weight, mature bolls were taken from each variety and weight of collected bolls was measured with the help of electric weighing balance in grams.

Statistical analysis: Univariate data analysis techniques are still being used while living in the age of multivariate (GrayMcGuire et al., 2009) where it is irrational to deal with different traits individually. The conventional technique of performing separate ANOVA for each trait not only ignores any relationships among the traits but also increases the Type I error rate which can result in erroneous and misleading conclusions. Multivariate Analysis of Variance (MANOVA) is the multivariate generalization of univariate ANOVA and compares the vector of means for all response variables across treatments rather than comparing the means for single response variable across treatments as with the ANOVA method (Bray and Maxwell, 1985). MANOVA takes into account the relationships among the response variables by analyzing them simultaneously which results in statistically high power for MANOVA than its univariate counterpart ANOVA technique. MANOVA was implemented to test the significance of treatment vector means for all response variables. Beyond MANOVA, three legitimate contrasts between Bt versus Non-Bt varieties, among Bt varieties, and among Non-Bt varieties were performed to test the research oriented pre-planned comparisons. The Hypothesis-Error (HE) plot (Fox et al., 2007; Friendly, 2007) was developed for the visual inspection of MANOVA and different contrast hypotheses.

Linear Discriminant Analysis (LDA) is a multivariate technique developed by Fisher (1940) for group separation. LDA was used to determine which yield traits (especially plant height, number of sympodial and monopodial branches, boll weight, number of bolls per plant and 50\% open boll) discriminate between cotton varieties. The scores of first two discriminant functions, along with cotton varieties identifiers, were plotted in two dimensional graphs to get more insight into the varietal group segregation. The $95 \%$ confidence ellipses in this case circles of radius (Seal, 1964), where $n_{i}$ is the group size and $Z_{\alpha / 2}$ is the standard normal deviate at $\alpha$ level, for each cotton variety were mapped. These confidence ellipses could be used to declare the significance of different groups. Correlations of variables with discriminant functions were also computed by using the formula:

$$
\rho=\frac{\operatorname{diag}\left(\{\operatorname{diag}(\mathrm{S})\}^{\frac{-1}{2}}\right) \mathrm{Sa}}{\sqrt{\mathrm{a}^{\prime} \mathrm{Sa}}}
$$

where $\mathrm{a}$ is the vector of coefficients of the linear discriminant function, $\mathrm{S}$ is the pooled unbiased estimate of $\Sigma$ (matrix of error sum of squares and cross products), and diag $\{\operatorname{diag}(\mathrm{S})\}$ -2 is the diagonal matrix with diagonal elements as the reciprocal of the square root of diagonal elements of S (Timm, 1975). All computations were implemented in the Statistical Programming Language R (Team, 2014).

\section{RESULTS}

MANOVA revealed that all the genotypes ( $\mathrm{Bt}$ and Non-Bt) and both varietal groups differ significantly with each other. Moreover, all the genotypes within their respective group were found significantly different (Table 2).

The data were subjected to linear discriminant analysis where first two linear discriminants explained 61.05 and $23.54 \%$ variability, respectively (Table 3). First linear discriminant revealed that plant height explained $72 \%$ variability due to disease development followed by $34 \%$ of $50 \%$ open bolls, $12 \%$ boll weight, $11 \%$ monopodial branches, $6 \%$ number of bolls and $4 \%$ sympodial branches, respectively. Similarly, the second linear discriminant exhibited the major contribution $63 \%$ of $50 \%$ open bolls followed by $52 \%$ number of bolls, $45 \%$ plant height, $39 \%$ boll weight, $35 \%$ sympodial branches and $9 \%$ monopodial branches, respectively. Plant height and $50 \%$ open bolls had relatively high correlation in both discriminants.

The means for two discriminants were plotted on the territorial map having confidence circles drawn around. Visual inspection revealed that disease influenced the yield traits (comprising plant height and $50 \%$ open bolls) of $\mathrm{Bt}$

Table 2. Multivariate analysis of variance depicting the comparison of different cotton genotypes according to their groups and within the respective groups for yield traits.

\begin{tabular}{llrrrrrr}
\hline Source of variation & Df & Wilks & F & df1 & df2 & P-value \\
\hline Variety & & 11 & 0.0564 & 5.999 & 66 & 556.90 & 103.00 \\
& Bt Vs Non-Bt & 1 & 0.6584 & 8.903 & 6 & 40.001 \\
& Among Bt & 7 & 0.1073 & 7.062 & 42 & 296.56 & $<0.001$ \\
& Among Non-Bt & 3 & 0.5598 & 3.690 & 18 & 291.81 & $<0.001$ \\
Residual & & 108.00 & & & & \\
\hline
\end{tabular}


cotton genotypes potentially than the Non-Bt genotypes as the confidence circles of $\mathrm{Bt}$ genotypes were scattered while the Non-Bt genotypes were clustered (Fig. 1).

Table 3. Linear discriminants revealing the variability in yield traits of different cotton genotypes.

\begin{tabular}{lcc}
\multicolumn{3}{c}{ yield traits of different cotton genotypes. } \\
\hline Traits & $\begin{array}{c}\text { First Linear } \\
\text { discriminant }\end{array}$ & $\begin{array}{c}\text { Second Linear } \\
\text { discriminant }\end{array}$ \\
\hline Plant Height & 0.72 & -0.45 \\
No. of Bolls & 0.06 & -0.52 \\
50\% Open Bolls & -0.34 & -0.63 \\
Boll weight & 0.12 & -0.39 \\
Sympodial & 0.04 & -0.35 \\
Monopodial & 0.11 & 0.099 \\
\hline
\end{tabular}

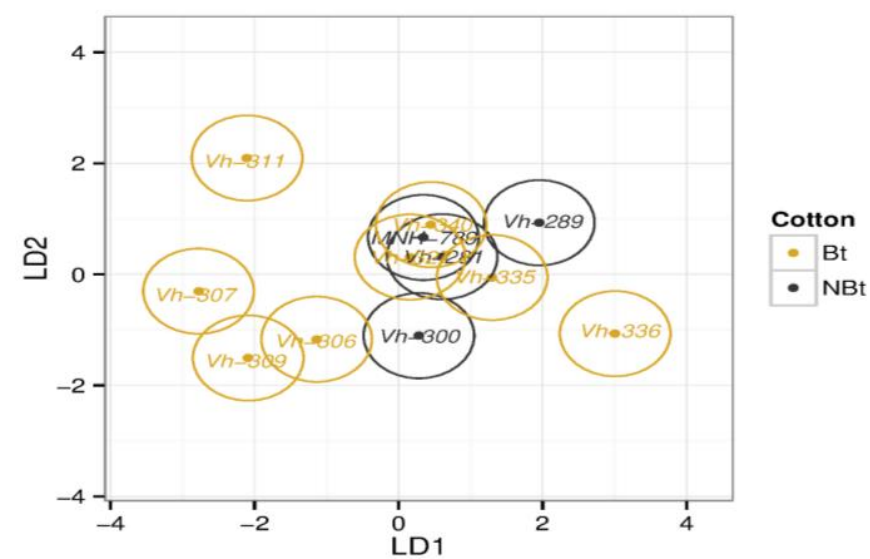

Figure 1. Territorial map of linear discriminant analysis depicting the visual comparison of $\mathrm{Bt}$ and NonBt cotton genotypes through the overlapping of confidence circles.

For instance, Bt genotypes encompassing Vh-336 and Vh-311 were far apart from the other's territorial map, hence revealing that their yield traits were influenced significantly in a different way than the other genotypes (Fig. 1). However, there was a little overlapping between Vh-307 and Vh-309, but their yield traits were inclined to disease development less than Vh-311 and Vh-336, and greater than remaining Bt genotypes. Since, the confidence circles of genotype Vh-306 and Vh-309 were overlapping; their yields traits were influenced equally with the disease development. Similarly, Vh-335, Vh-327 and Vh-340 didn't differ as their circles overlap each other (Fig. 1). Though, confidence circles of Non-Bt genotypes were gathered near to each other but Vh289 and Vh-300 differ significantly (both circles are far apart) from each other, while Vh-281 and MNH-789 do not differ (overlapping circles) with each other but the impact of disease on their yield traits differ significantly than Vh-289 and Vh300 (Fig. 1).

Additionally, the territorial map explained some other useful information that genotypes encompassing Vh-336, Vh-307,
Vh-289 and Vh-309 had a major contribution in $\mathrm{LD}_{1}$ while Vh-311, Vh-309 and Vh-336 had in LD $_{2}$. Most overlapping circles had score means round 0 in both discriminants and these genotypes were also non-significant.

Moreover, Heplot constructed between plant height and 50\% open bolls demonstrated negative correlation between the above described traits as the direction of ellipses (made for each contrast to investigate the impact of disease on yield traits of different genotypes from different groups) indicated. The $95 \%$ confidence ellipse naming variety indicated diverse impact of disease on yield traits of test genotypes. For instance, the genotypes including Vh-307, Vh-311 and Vh336 were outside the variety ellipse indicating that the yield traits of these genotypes were seriously affected by disease than other genotypes. Second ellipse naming among $\mathrm{Bt}$ explained that the yield traits of Bt genotypes comprising Vh307, Vh-309, Vh-311 and Vh-336 were impacted greatly than other Bt genotypes as these genotypes were lying outside among Bt ellipse. The remaining Bt genotypes were lying inside the among Bt ellipse therefore their means were nonsignificantly different (Fig. 2). Third ellipse of among Non$\mathrm{Bt}$ revealed that yield traits of Non-Bt genotypes including Vh-289 and Vh-300 were significantly influenced by disease (as these genotypes were outside the ellipse) than Vh-281 and MNH-789 (remained inside the ellipse). Bt vs Non Bt contrast was of one degree of freedom henceforth it degenerated to a line (Fig. 2). Heplots made by other pairs of genotypes didn't show such an exact pattern (not shown). These results were in conformation with those obtained by territorial map.

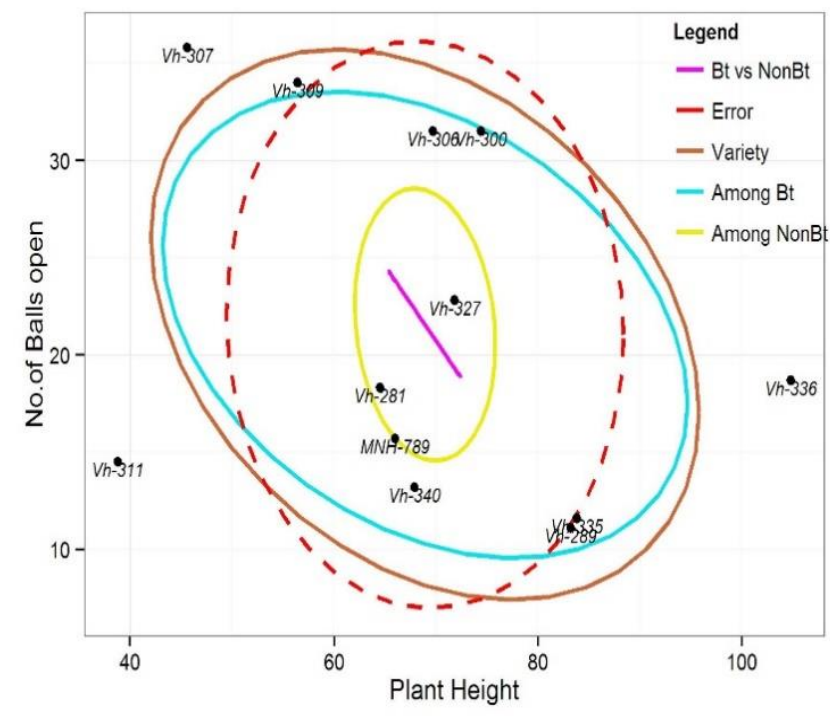

Figure 2. HE plot of Plant height and 50\% open boll's data depicting the variety (maroon), among Bt (blue), among Non-Bt (yellow) and error (red dotted) ellipses giving the visual estimate of difference between the genotypes regarding the plant height and $50 \%$ open bolls. 


\section{DISCUSSION}

The results of this study confirm earlier reports (Rashida et al., 2005; Ahmad et al., 2008; Akhtar et al., 2010) that cotton genotypes (Bt and Non-Bt) responded diversely to CLCuD which has emerged as a major threat to cotton production in Pakistan. Considerable variation in responses was observed when using whitefly and graft transmission to inoculate Gossypium genotypes with CLCuV in field and greenhouse experiments. Under field evaluation, all cultivated genotypes of Gossypium hirsutum and three genotypes of G. barbadense were susceptible while eleven genotypes that represented six wild and cultivated Gossypium species were considered to be highly resistant as they were free from infection (Akhtar et al., 2010). Sixty four cotton varieties/lines were screened against $\mathrm{CLCuV}$ disease; results revealed that Ravi and FDH 170 were highly resistant, 15 were resistant, and 12 were moderately resistant; whereas, nine varieties were found moderately susceptible, 19 were susceptible and seven were found highly susceptible to $\mathrm{CLCuV}$ disease (Rashida et al., 2005). Yield traits encompassing plant height and 50\% open bolls experienced major impact of disease development in case of Bt cotton than non-Bt cotton. The results demonstrated by Heplot constructed between plant height and $50 \%$ open bolls indicate that CLCuD has adverse impact on yield and fibre quality of cotton as it caused $52.7 \%$ reduction in number of bolls and $54.2 \%$ in boll weight in Bt cotton hybrid RCH 134. Similarly, the disease reduced the boll number and boll weight by 46.1 and $43.4 \%$, respectively in another Bt cotton hybrid MRC 6304 (Singh et al., 2013). Mahoomad et al. (1995) reported that in cotton cultivars the average reduction in plant height $40.6 \%$, boll weight $33.8 \%$, number of bolls per plant $72.5 \%$, ginning out turn $3.9 \%$, fibre length $3.4 \%$ and fibre strength $0.7 \%$ due to cotton leaf curl virus disease because of change in composition of major fiber components including cellulose, protein, wax and pectin (Farooq et al., 2013). But in view of Idris (1990) virus has significant impact on yield but not on fiber quality. Russel (1982) found that boll weight was negatively affected by $\mathrm{CLCuV}$. In another study, yield and yield-components and fiber traits of different genotypes/varieties (MNH-786, MNH789, MNH- 6070, CIM-496, and BH-160) were compared under epidemic conditions of CLCuD and results revealed significant differences in plant height, no. of bolls $/ \mathrm{m}^{-2}$, seedcotton yield (kg/ha) (Ahmad et al., 2008).

Thirty one cotton cultivars were tested under CLCuV intensive conditions to determine genotypic, phenotypic correlation coefficients using path analysis at the genotypic level between seed cotton yield, earliness, fiber and yield contributing traits. Heritability (broad sense) revealed higher estimates of fiber strength (97\%), CLCuV\% (97\%), fiber fineness $(91 \%)$, yield $\mathrm{kg} / \mathrm{ha}(91 \%)$, boll weight $(90 \%)$, plant height $(87 \%)$, bolls per plant $(86 \%)$, days taken to $1^{\text {st }}$ flower (84\%), Days taken to $1^{\text {st }}$ bud (82\%) while for nodes to $1^{\text {st }}$ fruiting branch $(56 \%)$, staple length $(46 \%)$, monopodia per plant $(42 \%)$ moderate but for GOT\% (32\%) and sympodia per plant (28\%) low estimates were found (Farooq et al., 2013). According to these researchers the traits like plant height, bolls per plant and sympodia per plant may be considered for selection in virus intensive conditions as they showed higher estimates of broad sense heritability along with positive and significant genotypic correlation with seed cotton yield. However, severely affected genotypes by CLCuV can be managed with increasing plant population and nitrogen fertilizer to achieve optimum seed-cotton yield (Ahmad et al., 2008).

Conclusion: It can be deduced that both statistical tools (Linear Discriminant Analysis (LDA) and Hypothesis-error (HE) plot) revealed similar results and explicitly highlighted the impact of the disease on cotton genotypes (Bt and non-Bt) and yield traits as well. The tools also explained severely affected yield traits in the study i.e. plant height and 50\% open bolls. Hence, plant pathologists, plant disease epidemiologists and plant breeders can use these tools potentially for selection of lines/varieties either for future breeding program or farmer field. Moreover, particularly affected yield traits by disease development can be addressed dynamically.

Acknowledgment: The author expresses thanks for the provision of experimental facilities especially seed from Cotton Research Institute, Vehari, and land and other services from Department of Plant Pathology, University of Agriculture, Faisalabad, Pakistan.

\section{REFERENCES}

Ahmad, G., S.A. Malik, Z. Mahmood, M.Z. Iqbal, S. Ahmad and S. Ahmad. 2002. Effect of cotton leaf curl virus disease on morphology, yield and fibre characteristics of susceptible lines/cultivars of cotton (Gossypium hirsutum L.). Asian J. Plant Sci. 1:705-707.

Ahmad, S., K. Hayat, F. Ashraf and M.A. Sadiq. 2008. Effect of cotton leaf-curl virus on the yield-components and fibre properties of cotton genotypes under varying plant spacing and nitrogen fertilizer. Sci. Technol. Develop. 27:33-41.

Ahmed, Z. 1999. Prospects and bottlenecks of cotton crop in Pakistan. Pak. Cotton Grower 3:6-7.

Akhtar, K.P., S. Haidar, M.K.R. Khan, M. Ahmad, N. Sarwar, M.A. Murtaza and M. Aslam. 2010. Evaluation of Gossypium species for resistance to cotton leaf curl Burewala virus. Ann. Applied Biol. 157:135-147.

Akhtar, K.P., M. Hussain, A.I. Khan, M.A. Haq and M.M. Iqbal. 2004. Influence of plant age, whitefly population and cultivar resistance on infection of cotton plants by cotton leaf curl virus (CLCuV) in Pakistan. Field Crops Res. 86:15-21. 
Akhtar, K.P., A.I. Khan and M.S.I. Khan. 2002. Improved bottle shoot grafting technique/method for the transmission of cotton leaf curl virus $(\mathrm{CLCuV})$. Nucleus 39:115-117.

Bray, J.H. and S.E. Maxwell. 1985. Multivariate analysis of variance Sage. University paper Series on Qualitative Research Methods, Vol. 24. Sage Publications, Newbury Park, California.

Briddon, R., S. Mansoor, I. Bedford, M. Pinner, K. Saunders, J. Stanley, Y. Zafar, K. Malik and P. Markham. 2001. Identification of DNA components required for induction of cotton leaf curl disease. Virol. 285:234-243.

Briddon, R.W. and P.G. Markham. 2000. Cotton leaf curl virus disease. Virus Res. 71:151-159.

Brown, J.K. 2001. Viral and phytoplasma disease: Cotton leaf curl disease, pp.52-54. In: T.L Kirkpatrick and C.K. Rothrock (eds.), Compendium of Cotton Disease, $2^{\text {nd }} \mathrm{Ed}$. American Phytopathological Society, St. Paul, Minnesota.

Dewey, D.R. and K. Lu. 1959. A correlation and pathcoefficient analysis of components of crested wheatgrass seed production. Agron. J. 51:515-518.

Farooq, J., M. Anwar, M. Riaz, A. Mahmood, A. Farooq, M.S. Iqbal and M.S. Iqbal. 2013. Association and path analysis of earliness yield and fiber related traits under cotton leaf curl virus $(\mathrm{CLCuV})$ intensive conditions in Gossypium hirsutum L. Plant Knowl. J. 1:43-50.

Fisher, R.A. 1940. The precision of discriminant functions. Ann. Eugen. 10:422-429.

Fortucci, P. 2002. The contribution of cotton to economy and food security in developing countries, pp.8-9. In: Cotton and Global Trade Negotiations sponsored by the World Bank and ICAC. Conference 8 July, 2002.

Fox, J., M. Friendly and G. Monette. 2007. Visual hypothesis tests in multivariate linear models: The heplots package for R. In DSC 2007: Directions in Statistical Computing. Auckland, NZ.

Friendly, M. 2007. HE plots for multivariate linear models. J. Comp. Graph. Stat. 16: 421-444.

Gray-McGuire, C., Y. Song, N.J. Morris and C.M. Stein. 2009. Comparison of univariate and multivariate linkage analysis of traits related to hypertension. BMC Proceedings. 3(Suppl 7):S99.

Haase, R.F. and M.V. Ellis. 1987. Multivariate analysis of variance. J. Counseling Psychol. 34:404-413.

Harrison, B.D., Y.L. Liv, S. Khalid, S. Hameed, G.W. Otim Nape and D.J. Robinson. 1997. Detection and relationship of cotton leaf curl virus and allied white fly transmitted Gemini viruses accruing in Pakistan. Ann. Appl. Biol. 130:61-75.

Idris, A.M. 1990. Cotton leaf curl virus disease in the Sudan. Mededelingen van de Faculteit Landbouwwetenschappen, Rijksuniversiteit. Gent. 55:263-267.

Khan, M.A. and H.A. Khan. 2000. Cotton leaf curl virus disease severity in relation to environmental conditions. Pak. J. Biol. Sci. 3:1688-1690.

Maas, S. 2013. Australian Cotton Production Manual. Cotton Research and Development Corporation, Narrabri, Australia.

Mahmood, T., M. Arshad, M. Tahir and M. Afzal. 1995. Response of some cotton varieties to cotton leaf curl virus. Pak. J. Sci. Indust. Res. 38:30-32.

Mughal, S.M. and M.A. Khan. 2006. Laboratory Manual for Plant Virology, p.125. Department of Plant Pathology, University of Agriculture, Faisalabad, Pakistan.

Rashida, P., M.K. Sultan, M.A. Khan and N.U. Islam. 2005. Screening of cotton germplasm against cotton leaf curl Begomovirus (CLCuV). J. Agri. Social Sci. 1:235-238.

Russel, T.E. 1982. Effect of cotton leaf curl crumple disease (CLC) on stab and planted cotton, p. 56. Bull. Agric. Exp. Stn. University of Arizona. Tucson, Arizona.

Seal, H.L. 1964. Multivariate statistical analysis for biologists. J. Wiley, Methuen, London.

Singh, D., J.S. Gill, R.K. Gumber, R. Singh and S. Singh. 2013. Yield and fibre quality associated with cotton leaf curl disease of Bt-cotton in Punjab. J. Environ Biol. 34:113-116.

Team, R.C. 2014. R: A language and environment for statistical computing. R. Foundation for Statistical Computing, Vienna, Austria. Available online at http://www.R-project.org/.

Timm, N.H. 1975. Multivariate analysis with Applications in education and Psychology. Brooks/Cole Pub. Co., Monetery California.

USDA, GAIN. 2016. Pakistan cotton: annual production and products. Foreign Agricultural Services. Availableat:http://gain.fas.usda.gov/Recent\%20GAIN\% 20Publications/Cotton\%20and\%20Products\%20Annual _Islamabad_Pakistan_4-1-2015.pdf.

Weinfurt, K.P. 1995. Multivariate analysis of variance, p.245276. In: L.G. Grimm and P.R. Yarnold (ed.), Reading and understanding multivariate statistics. American Psychological Association. Washington, DC. 